\title{
Amélioration génétique des performances zootechniques du porc local du Bénin par croisement avec le Large White
}

\author{
A. K. I. YOUSSAO, G. B. KOUTINHOUIN, T. M. KPODEKON, A. YACOUBOU, \\ A. G. BONOU, A. ADJAKPA, S. AHOUNOU et R. TAIWO
}

\author{
Ecole Polytechnique d'Abomey-Calavi, Département de Production et Santé Animales, \\ 01 BP 2009, Cotonou, Bénin. \\ *Auteur correspondant, E-mail : issaka.youssao@epac.uac.bj
}

\begin{abstract}
RESUME
Le but de cette étude est d'améliorer les performances zootechniques des porcs locaux du Bénin par croisement avec le Large White. Au total, 4 lots de porcelets ont été constitués : porcs locaux, Large White, croisés mâle Large White et femelles locales (MLW x FL) et enfin, croisés mâle local et femelles Large White (ML x FLW). Cent sept porcelets ont été obtenus et élevés dans les mêmes conditions pendant 3 mois. Les truies de race locale ont une taille moyenne de portée faible $(\mathrm{P}<0,01)$ par rapport aux truies de race Large

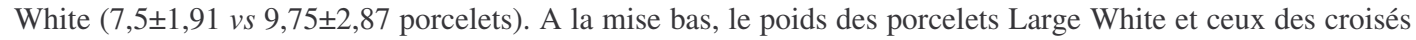
ont été significativement plus élevés que ceux des porcelets locaux $(\mathrm{P}<0,01)$. A la fin de l'expérimentation, les croisés ont un poids supérieur à celui des porcs locaux $(\mathrm{P}<0,001)$ et inférieur à celui des Large White $(\mathrm{P}<0,001)$. L'hétérosis a été positif pour la taille de la portée lorsque le mâle Large White est utilisé comme géniteur (28\%). Par contre, lorsque le porc local a été utilisé comme géniteur, un hétérosis négatif a été obtenu pour les taux de mortinatalité et de mortalité naissance-sevrage.
\end{abstract}

(c) 2009 International Formulae Group. All rights reserved.

Mots clés : Porc local, Large White, métis, croissance, poids, hétérosis.

\section{INTRODUCTION}

Le porc local béninois ou «porc nain de l'Afrique de l'Ouest », se rencontre le long des pays côtiers de la sous région Ouestafricaine et descend probablement du porc européen (Devendra et Fuller, 1979; d'Orgeval, 1997). Sur le plan phylogénétique, il est proche des sangliers italiens et turques, des porcs ibérique, espagnol et belge (Ramirez et al., 2006). Le porc local du Bénin présente des haplotypes E1 et dénote une introgression de souches européennes comme le Large White et de souches asiatiques (Ramirez et al., 2006).

Sur le plan ethnologique, le groin du porc local est long, cylindrique, conique et effilé à l'extrémité. Son profil est légèrement concaviligne et ses oreilles sont petites, dressées et parfois rejetées en arrière. Ils sont de petit format avec une hauteur au garrot de
$40 \mathrm{~cm}$, hauts sur pattes, un corps étroit de 60 $\mathrm{cm}$ de longueur et une cuisse plate avec un jambon peu fourni. Son poids adulte varie entre 40 et $50 \mathrm{~kg}$. Le phénotype dominant est la robe noire avec des extrémités ou ceinture blanche. On y rencontre également les robes blanches avec des extrémités noires ou grises.

Le porc local béninois présente des performances de croissance modeste, une faible productivité numérique mais il est caractérisé par une grande rusticité. L'âge à la première saillie est de 6 mois avec une portée moyenne de 6,86 porcelets (Youssao et al., 2009). Youssao et al. (2009) rapportent des gains quotidiens moyens en engraissement de $126,4 \mathrm{~g} / \mathrm{j}$ pour les femelles et $74 \mathrm{~g} / \mathrm{j}$ pour les mâles en station contre $60 \mathrm{~g} / \mathrm{j}$ enregistré dans les élevages traditionnels (Atodjinou et Dotcho, 2006). Ces performances ne sont pas encore satisfaisantes malgré plusieurs études 
visant à améliorer son alimentation et les conditions de son élevage (d'Orgeval, 1997 ; Agbokounou, 2001 ; Codjo, 2003). Les races exotiques telles que Landrace et Large White ont un gain quotidien moyen qui varie entre 600 et $900 \mathrm{~g} / \mathrm{j}$ et une taille moyenne de portée qui varie entre 10 et 12 pocelets (Youssao, 2007). Ces valeurs montrent que ces deux races présentent de meilleures performances par rapport à celles des races locales (Holnes, 1991). Cependant, la résistance de la race locale à certaines pathologies du porc est prouvée (Muys et al., 2003).

Hormis les effets que pourrait induire une alimentation adéquate, les performances des races locales pourront aussi être améliorées génétiquement par le croisement ou par la sélection. Parmi ces deux méthodes, seul le croisement permet d'obtenir un résultat immédiat. L'objectif de cette étude est d'évaluer les performances zootechniques des métis issus du croisement Large White et porc local par rapport à celles des parents et les effets hétérosis au niveau des métis.

\section{MATERIEL ET METHODES}

Site de l'étude et infrastructures d'élevage

Une étude sur l'amélioration génétique du porc de race locale a été réalisée du 5 août 2007 au 20 mai 2008 sur la ferme de la Production et Santé Animales (PSA) de l'Ecole Polytechnique d'Abomey-Calavi (EPAC). Elle est située dans le Département de l'Atlantique au Sud Bénin et bénéficie des conditions climatiques de type subéquatorial, caractérisées par deux saisons de pluies: la grande (avril à juillet) et la petite (septembre à novembre). Ces deux saisons sont intercalées par des saisons sèches. La pluviométrie moyenne avoisine $1200 \mathrm{~mm}$ par an. On y enregistre des températures mensuelles moyennes variant entre 27 et $31{ }^{\circ} \mathrm{C}$ et un taux d'humidité relative compris entre $65 \%$ (de janvier à mars) et $97 \%$ (de juin à juillet).

Les dimensions de la porcherie sont de $20 \mathrm{~m}$ de long et 4,5 m de large. Elle est de type semi-ouvert avec des murets de $1 \mathrm{~m}$ de haut, et subdivisée en huit loges de 11,25 $\mathrm{m}^{2}$ dont six ont été utilisées pour cette étude. Chaque loge est munie d'un abreuvoir et d'une mangeoire. La toiture est en tôles ondulées et le sol cimenté est en pente douce orientée vers les rigoles d'évacuation des urines.

\section{Animaux et mode d'élevage}

Les animaux utilisés dans cette expérimentation sont issus de parents de races Large White et porcs locaux, âgés de 13 mois \pm 26 jours. Quatre lots ont été constitués dont un premier composé d'un verrat Large White et de trois truies primipares Large White, le second est composé d'un verrat local et de trois truies locales ayant préalablement réalisées deux mises bas, le troisième est composé d'un verrat Large White et deux truies locales ayant préalablement réalisées deux mises bas (MLW x FL) et enfin le quatrième composé d'un verrat local et deux truies Large White primipares (ML x FLW).

Après la constitution des lots, les mâles ont été séparés des femelles. Les femelles ont été quotidiennement suivies pour détecter les périodes de chaleur. Chaque fois qu'une femelle en chaleur a été identifiée, elle était conduite dans le box du mâle pour la saillie. Après la saillie, la femelle a été reconduite dans son box initial. Elle a été remise au mâle si elle présente à nouveau des signes cliniques de chaleur.

Au cours de la gestation, les femelles ont été bien suivies sur les plans sanitaire et nutritionnel. Dès l'apparition des signes prémonitoires de la mise bas, la femelle a été retirée du lot et transférée à la maternité préalablement désinfectée. Cent sept porcelets ont été obtenus dont 30 de race locale, 39 Large White, 22 croisés MLW x FL et 16 ML $x$ FLW.

Les animaux ont été nourris avec un aliment composé de : son de blé (38\%), farine de poisson $(2 \%)$, son de maïs $(30 \%)$, tourteau de palmiste $(28 \%)$, phosphate bicalcique $(0,2 \%)$, lysine $(0,1 \%)$, méthionine $(0,1 \%)$, sel de cuisine $(0,5 \%)$ et coquille d'huitre $(1 \%)$. La quantité d'aliment servie a été de $2 \mathrm{~kg}$ par truie et par jour et dose $2900 \mathrm{kcal}$ et $16 \%$ de protéines brutes. L'eau a été servie ad libitum.

Pendant la durée de l'étude, tous les animaux ont été régulièrement suivis sur les plans sanitaire et médical. Les examens coprologiques ont été faits deux fois, à un intervalle de deux mois pour dépister les animaux infestés par les strongles digestifs chez les reproducteurs. Les prélèvements de sang ont été réalisés pour le dépistage des animaux infestés par les trypanosomes. Ceux qui sont malades sont systématiquement traités suivant les cas cliniques présentés. 
Deux pédiluves à but prophylactique ont été installés à l'entrée des locaux.

\section{Collecte des données}

A chaque mise bas, le nombre d'animaux par portée a été enregistré. Les morts nés ont été enregistrés et déduits de la portée. Les porcelets nés vivants ont été d'abord pesés entre deux à trois heures après la mise bas et ensuite tous les 15 jours, jusqu'à l'âge de 90 jours à l'aide d'une balance de capacité $10 \mathrm{~kg}$ et de $50 \mathrm{~g}$ de précision. Le nombre de porcelets morts entre la naissance et le sevrage a été également enregistré. Le sevrage a été fixé à 60 jours.

\section{Analyse statistique}

Le taux de mortinatalité (nombre de morts nés sur nés totaux), le taux de nés vivants (nombre de nés vivants sur le nombre de nés totaux), le taux de mortalité naissancesevrage (nombre de morts enregistrés de la naissance au sevrage sur nombre de nés vivants) et le taux de porcelets sevrés (nombre de porcs sevrés sur nombre de porcs nés vivants), ont été ensuite calculés. Le gain quotidien moyen a été également calculé par période de 15 jours.

Un modèle linéaire à effets fixes a été ajusté aux données de poids et de gain de poids et comprend les effets fixes du type génétique et du sexe. L'interaction entre le type génétique et le sexe a été significative et prise en compte dans le modèle d'analyse. Ce modèle se présente de la manière suivante :

Avec

$$
Y_{i j k}=\mu+T_{i}+S_{j}+T S_{i j}+e_{i j k}
$$

- $\mathrm{Y}_{\mathrm{ijk}}$ : le poids ou le gain moyen quotidien du $\mathrm{k}^{\mathrm{e}}$ porc, du type génétique i et du sexe $\mathrm{j}$;

- $\quad \mu$ : la valeur de la moyenne générale ;

- $\mathrm{T}_{\mathrm{i}}$ : effet fixe du type génétique $\mathrm{i}$ (porcs locaux, Large White, Métis MLW x FL et Métis MLx FLW) ;

- $S_{\mathrm{j}}$ : effet fixe du sexe $\mathrm{j}$ (femelle et mâle).

- $\mathrm{TS}_{\mathrm{ij}}$ : interaction entre type génétique $\mathrm{i}$ et sexe j de l'animal ;

- $\mathrm{e}_{\mathrm{ijk}}$ : effet résiduel aléatoire.

La procédure des modèles linéaires généralisés (Proc GLM) du SAS (Statistical Analysis System, 1991) a été utilisée pour l'analyse de la variance et les moyennes ont été ensuite calculées et comparées par le test de $t$.
L'hétérosis a été calculée par la formule suivante :

Hétérosis $=\frac{F_{1}-\frac{(\text { Père }+ \text { Mère })}{2}}{\frac{(\text { Père }+ \text { Mère })}{2}}$

Où :

- F1 est la valeur de la performance de l'individu issu du croisement LW-PL,

- Père, est la valeur de la performance du père de l'individu $\mathrm{F} 1$;

- Mère, est la valeur de la performance de la mère de l'individu F1.

Pour les taux de mortinatalité, de nés vivants, de mortalité naissance- sevrage et de porcelets sevrés, un intervalle de confiance à 95\% a été calculé suivant la formule cidessous :

$$
I C P=1,96 \sqrt{\frac{[P(1-P)]}{N}}
$$

Où, $\mathrm{P}$ est le taux et $\mathrm{N}$ la taille de l'échantillon La comparaison entre ces différents taux a été faite par la procédure Proc freq du SAS (1991) en utilisant le test de Chi-carré.

\section{RESULTATS \\ Productivité numérique}

Le tableau 1 présente les paramètres de productivité numérique des truies en fonction du mode de croisement. La taille de la portée a varié en fonction de type génétique et du sens de croisement $(\mathrm{P}<0,001)$. La taille de portée de la truie de race locale, est faible $(\mathrm{P}<0,01)$ par rapport aux truies de race Large White $(7,5 \pm 1,91$ vs $9,75 \pm 2,87$ porcelets $)$. A l'issue des mises bas, la taille de la portée la plus importante a été obtenue chez les femelles locales croisées avec les mâles Large White (11 \pm 1,41 porcelets). En croisant les femelles Large White au mâle local, la taille de la portée a été identique à celle des truies de race locale $(8 \pm 1,41$ porcelets). Aucune différence significative n'a été observée entre les taux de mortinatalité, de viabilité à la naissance et au sevrage et le taux de mortalité naissance-sevrage des porcelets croisés et des porcelets locaux et Large White. Toutefois, un taux de mortinatalité de $23 \%$ a été obtenu dans le croisement MLW x FL contre $17 \%$ et $10 \%$, respectivement chez les porcelets locaux et Large White. La plus faible valeur de mortinatalité $(6 \%)$ a été obtenue chez les croisés ML x FLW. Les taux de mortalité 
naissance-sevrage ont varié de $8,33 \%$ à $20 \%$ en fonction du type génétique, sans toutefois présenter de différences significatives.

\section{Performances de croissance}

Le tableau 2 présente les performances de croissance des porcelets par type génétique et en fonction de l'âge. Des variations pondérales à âge type selon le type génétique ont été observées $(\mathrm{P}<0,001)$. Les poids des porcelets Large White et ceux des croisés à la mise bas et à 1 mois d'âge ont été significativement plus élevés que ceux des porcelets locaux. Aucune différence n'a été observée entre les poids des porcelets Large White et ceux des croisés à l'âge d'1 mois. Par rapport aux porcelets locaux et aux croisés MLW x FL, les porcelets Large White ont les poids les plus élevés à deux mois d'âge $(\mathrm{P}<0,001)$. A la fin de l'expérimentation, le poids des porcelets Large White a été le triple de celui des porcelets locaux et les croisés ont un poids supérieur à celui des porcs locaux $(\mathrm{P}<0,001)$ et inférieur à celui des Large White $(\mathrm{P}<0,001)$. Parmi les croisés, les meilleurs poids ont été obtenus lorsque le mâle local est utilisé comme géniteur.

Le gain quotidien moyen a présenté des variations selon le type génétique $(\mathrm{P}<0,001)$. Les meilleurs gains ont été obtenus chez la race Large White, comparativement aux croisés et aux porcelets locaux $(\mathrm{P}<0,001)$.

\section{Effet du sexe et interaction entre sexe et type génétique}

A la naissance, le poids des mâles a été de $828 \mathrm{~g}$ et celui des femelles, $731 \mathrm{~g}$. A la fin de l'expérimentation, les poids ont été de 6591 et $6560 \mathrm{~g}$ respectivement pour les mêmes sexes. De la mise bas à 90 jours d'âge, le poids des femelles n'a pas été différent de celui des mâles pour l'ensemble des animaux (tous types génétiques confondus). L'interaction entre sexe et type génétique a été significative au niveau du poids à 90 jours d'âge et au niveau du gain quotidien moyen du troisième mois. Le tableau 3 présente l'effet de l'interaction entre type génétique et sexe sur les performances de croissance. Les femelles Large White ont un poids significativement plus important que les mâles de la même race (11786 g vs $9000 \mathrm{~g}$ ) tandis que les poids des mâles Large White sont plus élevés que ceux des deux types de croisés. Les mâles et les femelles F1 ont des poids identiques alors que les mâles de race locale ont été plus lourds $(\mathrm{P}<0,01)$ que les femelles de la même race. Le gain quotidien moyen, des femelles Large White a été meilleur à celui des mâles $(\mathrm{P}<0,01)$. Cependant, les mâles et les femelles des locaux et des croisés ont présenté des gains quotidiens moyens similaires.

\section{Effet hétérosis}

Pour les performances de reproduction, l'effet hétérosis est fortement influencé par le sens du croisement. Il a été positif pour la taille de la portée lorsque le mâle Large White est utilisé comme géniteur (28\%). Par contre, lorsque le porc local a été utilisé comme géniteur, un effet hétérosis négatif a été obtenu pour les taux de

Tableau 1: Productivité numérique des truies de race locale et Large White en fonction du mode de croisement.

\begin{tabular}{lcccc}
\hline Variables & Local $(\mathbf{N}=\mathbf{3 0})$ & $\begin{array}{c}\text { Large White } \\
(\mathbf{N}=\mathbf{3 9})\end{array}$ & $\begin{array}{c}\text { MLW X } \\
\text { FL }(\mathbf{N}=\mathbf{2 2})\end{array}$ & $\begin{array}{c}\text { ML X FLW } \\
(\mathbf{N}=\mathbf{1 6})\end{array}$ \\
\hline Taille moyenne de la portée & $7,5 \pm 1,9 \mathrm{a}$ & $9,7 \pm 2,8 \mathrm{~b}$ & $11 \pm 1,4 \mathrm{c}$ & $8 \pm 1,4 \mathrm{ab}$ \\
Taux de mortinatalité $(\%)$ & $16,67 \pm 13,3 \mathrm{a}$ & $10,26 \pm 9,5 \mathrm{a}$ & $22,73 \pm 17,5 \mathrm{a}$ & $6,25 \pm 11,8 \mathrm{a}$ \\
Nés vivants (\%) & $83,33 \pm 13,3 \mathrm{a}$ & $89,74 \pm 9,5 \mathrm{a}$ & $77,27 \pm 17,5 \mathrm{a}$ & $93,75 \pm 11,8 \mathrm{a}$ \\
Mortalité naissance - sevrage (\%) & $16,00 \pm 13,1 \mathrm{a}$ & $20,00 \pm 12,5 \mathrm{a}$ & $15,79 \pm 15,2 \mathrm{a}$ & $8,33 \pm 13,5 \mathrm{a}$ \\
Taux de sevrage (\%) & $84,00 \pm 13,1 \mathrm{a}$ & $80,00 \pm 12,5 \mathrm{a}$ & $84,21 \pm 15,2 \mathrm{a}$ & $91,67 \pm 13,5 \mathrm{a}$ \\
\hline
\end{tabular}

MLW X FL: mâle Large White X femelle locale; ML X FLW : mâle local X femelle Large White; les moyennes ou les taux de la même ligne suivis des lettres différentes diffèrent significativement au seuil de 5\%. Les moyennes sont suivies des écarts types et les pourcentages, des intervalles de confiance. 
Tableau 2 : Moyennes moindres carrés et erreurs standard des poids et gains quotidiens moyens des porcs locaux, Large White et issus des croisements MLW X FL et ML X FLW.

\begin{tabular}{lccccc}
\hline Variables & $\begin{array}{c}\text { Local } \\
(\text { moyenne } \pm \text { ES) }\end{array}$ & $\begin{array}{c}\text { Large White } \\
(\text { moyenne } \pm \text { ES })\end{array}$ & $\begin{array}{c}\text { MLW X FL } \\
(\text { moyenne } \pm \text { ES })\end{array}$ & $\begin{array}{c}\text { ML X FLW } \\
(\text { moyenne } \pm \text { ES) }\end{array}$ & $\begin{array}{c}\text { Test de } \\
\text { signification }\end{array}$ \\
\hline P0 $(\mathrm{g})$ & $570,7 \mathrm{~b} \pm 65,2$ & $886,4 \mathrm{a} \pm 50,0$ & $881,3 \mathrm{a} \pm 46,1$ & $852,1 \mathrm{a} \pm 89,6$ & $* *$ \\
P15 $(\mathrm{g})$ & $1252,5 \mathrm{c} \pm 194,1$ & $3056,8 \mathrm{a} \pm 143,3$ & $2088,5 \mathrm{~b} \pm 141,4$ & $2612,5 \mathrm{a} \pm 256,7$ & $* * *$ \\
P30 $(\mathrm{g})$ & $1883,3 \mathrm{~b} \pm 279,1$ & $4318,2 \mathrm{a} \pm 206,1$ & $3880,7 \mathrm{a} \pm 203,3$ & $4229,2 \mathrm{a} \pm 369,2$ & $* * *$ \\
P60 $(\mathrm{g})$ & $2301,1 \mathrm{~b} \pm 379,1$ & $7227,3 \mathrm{a} \pm 266,9$ & $6198,1 \mathrm{~b} \pm 263,4$ & $6808,3 \mathrm{ab} \pm 478,2$ & $* * *$ \\
P90 $(\mathrm{g})$ & $3353,7 \mathrm{~d} \pm 277,1$ & $10392,8 \mathrm{a} \pm 220,8$ & $5300,0 \mathrm{c} \pm 277,1$ & $7254,2 \mathrm{~b} \pm 315,5$ & $* * *$ \\
GMQ1 $(\mathrm{g} / \mathrm{j})$ & $43,6 \mathrm{~b} \pm 7,7$ & $114,4 \mathrm{a} \pm 5,7$ & $101,5 \mathrm{a} \pm 5,6$ & $112,6 \mathrm{a} \pm 10,3$ & $* * *$ \\
GMQ2 $(\mathrm{g} / \mathrm{j})$ & $23,2 \mathrm{c} \pm 9,4$ & $96,9 \mathrm{a} \pm 6,2$ & $77,2 \mathrm{~b} \pm 6,1$ & $85,97 \mathrm{ab} \pm 11,1$ & $* * *$ \\
GMQ3 $(\mathrm{g} / \mathrm{j})$ & $43,8 \mathrm{~b} \pm 8,6$ & $82,1 \mathrm{a} \pm 6,3$ & $11,7 \mathrm{c} \pm 14,3$ & $14,9 \mathrm{c} \pm 8,9$ & $* * *$ \\
\hline
\end{tabular}

**: $\mathrm{P}<0,01 ; * * *: \mathrm{P}<0,001 ; \mathrm{Pi}$ : Poids à i jour; ES : erreur standard; GMQi : Gain moyen quotidien du ième mois ; les moyennes suivies de la même lettre ne diffèrent pas significativement au seuil de 5\%; MLW X FL : mâle Large White X femelle locale ; ML X FLW : mâle local X femelle Large White.

mortinatalité et de mortalité naissancesevrage. Les valeurs des hétérosis pour les paramètres de productivité numérique sont données au tableau 4.

Pour le poids, la valeur de l'effet hétérosis a variée de $-0,06$ à $0,48 \%$ avec une moyenne de $27 \%$ chez les mâles et $17 \%$ chez les femelles issues du croisement MLW et FL. Dans le cas du croisement ML et FLW, l'effet hétérosis moyen a été de $18,32 \%$ chez le mâle et $5,25 \%$ chez les femelles. Les tableaux 5 et 6 présentent les effets hétérosis des mâles et des femelles issus des différents croisements.

\section{DISCUSSION}

\section{Productivité numérique \\ Taille de la portée}

La truie de race locale a une taille de portée faible par rapport aux truies de race Large White. A l'issue des croisements, la taille de la portée la plus importante a été obtenue chez les femelles locales croisées avec le mâle Large White. En croisant les femelles Large White au mâle local, la taille de la portée a été identique à celle des truies de race locale. Il apparaît donc que le croisement entre le porc de race locale et la Large White a amélioré la taille de la portée lorsque le mâle Large White est utilisé comme géniteur. La différence entre ces deux types de croisement serait due à la composante maternelle qui donne la supériorité du type de croisement. Au Sud du Bénin, la taille de la portée est de 7,3 chez les porcs locaux et varie de 8,9 à 9,6 chez les porcs exotiques élevés en milieu tropical (d'Orgeval, 1997). Les tailles de la portée obtenues au cours de la présente étude sont proches de celles de d'Orgeval (1997) et pourront s'améliorer au cours des mises bas suivantes dans la mesure où la taille de la portée s'améliore en fonction du rang de mise bas et que les truies Large White utilisées au cours de cette étude étaient à leur première portée. A la ferme d'élevage de Kpinnou où le mode d'élevage des porcs est similaire à celui de la présente étude, la taille moyenne des portées est de 6,31, le nombre moyen de nés vivants est de 5,74 chez les porcs locaux et chez les croisés elles sont respectivement de 6,86 et 6,20 (Bonou, 2006). Les tailles de la portée des Large White et des croisés obtenues dans la présente étude ont été meilleures que celles de la Ferme de Kpinnou. Mortalité

Les croisements réalisés dans la présente étude n'ont pas affecté les mortalités. Des taux variant entre 15 et $19 \%$ chez les porcs locaux et entre 15 à $28 \%$ chez les croisés et les Large White ont été rapportés par d'Orgeval (1997) alors que dans la présente étude, des taux variant de 8 à $20 \%$ ont été obtenus. Dans les élevages traditionnels au Sud du Bénin, 21,74\% de cas de mortalité ont été observés de la naissance au sevrage (Atodjinou et Dotcho, 2006). Mais ce taux était très faible $(9,05 \%)$ à la Ferme de Kpinnou pour les animaux bénéficiant de meilleures conditions d'élevage. De manière générale, les taux de mortinatalité et de mortalité des porcs sont globalement élevés et une étude des causes de morbidité et de 
mortalité serait nécessaire pour améliorer la viabilité des animaux.

\section{Performances pondérales}

A la mise bas et à un mois d'âge, le poids des porcelets Large White et ceux des croisés ont été significativement plus élevés que ceux des porcelets locaux. Contrairement aux résultats obtenus dans la présente étude, d'Orgeval, (1997) n'a pas obtenu une différence entre le poids à la naissance des races exotiques en milieu tropical et celui des races locales en station et même en élevage villageois. Dans le cas de la présente étude, les poids à la naissance ont été pris dans l'intervalle de deux à trois heures après la mise bas alors que dans les autres études, le poids à la mise bas a été pris entre 1 à 4 jours après la mise bas pour les élevages traditionnels de porcs locaux (d'Orgeval, 1997 ; Atodjinou et Docho, 2006), le jour de la mise bas chez les porcs exotiques en élevage semi amélioré. A la Ferme de Kpinnou, où les porcelets sont pesés quelques heures après la mise bas, le poids moyen des porcelets locaux est de $510 \mathrm{~g}$ et est similaire aux résultats de la présente étude (Bonou, 2006). Toutefois, chez les métis, le poids moyen est de 1044 g à la Ferme de Kpinnou, contre 852 à $881 \mathrm{~g}$ dans la présente étude. La différence entre ces deux poids est liée aux races utilisées dans le schéma de croisement. A la Ferme de Kpinnou, les métis proviennent d'un croisement Large White et Landrace alors que dans le cas de la présente étude, il s'agit d'un croisement Large White et porc local.

Au sevrage réalisé à deux mois d'âge, les Large White avaient un poids de $7227 \mathrm{~g}$ et les locaux, $2300 \mathrm{~g}$ tandis que les croisés avaient un poids intermédiaire de 6198 et $6808 \mathrm{~g}$, respectivement pour les croisements MLW x FL et ML x FLW. A la Ferme de Kpinnou, le poids au sevrage est de 2550 g, correspondant à un âge moyen de 58 jours (Bonou, 2006). Il en ressort que les porcelets locaux sevrés à la ferme de l'EPAC ont un poids identique à ceux de Kpinnou. Le croisement améliore le poids des descendants de plus de $60 \%$ par rapport à la race pure locale. Il est important de souligner que les quantités d'aliments consommés sont les mêmes, mais la valorisation de ces aliments en terme de croissance s'exprime de manière différente.

\section{Effet hétérosis}

Les croisements entre le porc Large White et les porcs locaux ont donné des métis dont les effets hétérosis ont été observés au niveau des performances pondérales et des performances de reproduction. Il a été donc observé que l'expression du génotype des individus de la première génération (F1) a été supérieure à la moyenne des valeurs des performances des parents, contrairement à ce qui devrait se produire s'il y avait simple addition de caractères. Le croisement réduit la fréquence des allèles homozygotes (couples de gènes identiques) et augmente le nombre d'allèles hétérozygotes (Bonnes et al., 1991). De ce fait, la plupart des caractères récessifs, défavorables, sont masqués par des caractères dominants, favorables. C'est pourquoi les métis ont souvent des capacités génétiques nettement supérieures à la moyenne de celles de leurs parents. Dans la présente étude, les valeurs des hétérosis sont élevées pour le poids et s'expliquent par l'éloignement de ces deux races sur le plan génétique.

Dans le cadre de l'estimation de l'effet de la race et de l'hétérosis sur les performances de croissance, l'effet de l'hétérosis individuel est de 8,1\%, 7,2\%,3\% et $11 \%$, respectivement pour les poids à la naissance, à 14 , à 28 et à 56 jours, si les croisements sont réalisés sur des races porcines voisines, notamment, le Yorkshire, le Landrace, le Large White et Chester White (Cassady et al., 2002). Ces effets augmentent lorsque les croisements se font entre races génétiquement différentes (Hampshire, Duroc, Piétrain et Spot), avec des hétérosis de 10,9; 16,$2 ; 14,4$ et $19,8 \%$, respectivement pour les poids à la naissance, à 14 , à 28 et à 56 jours (Cassady et al., 2002).

L'effet hétérosis a été positif pour la taille de la portée lorsque le mâle Large White est utilisé comme géniteur. Par contre, lorsque le porc local a été utilisé comme géniteur, un effet hétérosis négatif a été obtenu pour les taux de mortinatalité et de mortalité naissance-sevrage. Ces différences sont ainsi dues à la composante maternelle qui donne la supériorité du type de croisement. Les effets hétérosis sont plus importants pour les performances de reproduction et s'expliquent 
A. K. I. YOUSSAO et al. / Int. J. Biol. Chem. Sci. 3(4): 653-662, 2009

Tableau 3: Interaction entre type génétique et sexe sur les poids et les GQM des porcs locaux, Large White et issus des croisements MLW X FL et ML X FLW.

\begin{tabular}{|c|c|c|c|c|c|c|c|c|c|c|}
\hline \multirow{2}{*}{ Variables } & \multicolumn{2}{|c|}{ Local } & \multicolumn{2}{|c|}{ Large White } & \multicolumn{2}{|c|}{ MLW X FL } & \multicolumn{2}{|c|}{ ML X FLW } & \multirow[t]{2}{*}{ DSR } & \multirow{2}{*}{$\begin{array}{c}\text { Test de } \\
\text { signification }\end{array}$} \\
\hline & Mâle & Femelle & Mâle & Femelle & Mâle & Femelle & Mâle & Femelle & & \\
\hline $\mathrm{P} 0(\mathrm{~g})$ & $650,0 \mathrm{~b}$ & $491,4 b$ & $845,4 a$ & $927,3 a$ & $883,3 \mathrm{ab}$ & $739,3 \mathrm{ab}$ & $937,5 a$ & $766,67 \mathrm{ab}$ & 234,57 & NS \\
\hline P15 (g) & $1605,1 b c$ & $900,11 \mathrm{c}$ & $2802,2 \mathrm{a}$ & $3313,6 \mathrm{a}$ & $2235,1 \mathrm{a}$ & $1941,9 a$ & $2675 a$ & $2550 \mathrm{a}$ & 672,24 & NS \\
\hline P30 (g) & $2533,3 c$ & $1233,3 d$ & $4181,8 \mathrm{a}$ & $4454,6 a$ & $4011,0 \mathrm{ab}$ & $3761,5 b$ & $4375 \mathrm{ab}$ & $4083,33 \mathrm{ab}$ & 966,77 & NS \\
\hline P60 (g) & $2801,1 \mathrm{c}$ & $1800 \mathrm{c}$ & $7000 \mathrm{a}$ & $7454,6 a$ & $6150,6 b$ & $6246,2 b$ & $7250 \mathrm{a}$ & $6366,67 \mathrm{ab}$ & 1252,21 & NS \\
\hline P90 (g) & $4187,5 \mathrm{e}$ & $2520 \mathrm{f}$ & $9000 \mathrm{~b}$ & $11785,7 \mathrm{a}$ & $5409,1 \mathrm{~d}$ & $5201,0 d$ & $7775 c$ & $6733,33 c$ & 826,04 & $* * *$ \\
\hline GMQ1 (g/j) & $62,78 b$ & $24,33 \mathrm{c}$ & $111,21 \mathrm{a}$ & $117,6 \mathrm{a}$ & $102,3 a$ & $100,6 a$ & $114,6 \mathrm{a}$ & $110,6 a$ & 26,8 & NS \\
\hline GMQ2 (g/j) & $27,5 \mathrm{c}$ & $18,89 \mathrm{~d}$ & 93,94 & $100,0 \mathrm{a}$ & $71,7 \mathrm{~b}$ & $82,8 \mathrm{ab}$ & $95,8 \mathrm{ab}$ & $76,1 \mathrm{ab}$ & 29,1 & NS \\
\hline GMQ3 (g/j) & $55,56 \mathrm{~b}$ & $32,01 b$ & $52,38 \mathrm{~b}$ & $111,9 a$ & $6,7 b$ & $16,7 b$ & $17,5 \mathrm{~b}$ & $12,2 b$ & 23,4 & $* *$ \\
\hline
\end{tabular}

NS : P $>0,05 ; * *: \mathrm{P}<0,01 ; * * *: \mathrm{P}<0,001 ; \mathrm{Pi}:$ Poids à i jour; DSR : déviation standard résiduelle; GMQi : gain moyen quotidien du ième mois ;MLW X FL : mâle Large White X femelle locale; ML X FLW : mâle local X femelle Large White.

Tableau 4 : Effet hétérosis des paramètres de productivité numérique chez les porcs issus des croisements MLW X FL et ML X FLW.

\begin{tabular}{lccccccc}
\hline Variable & Local & Large White & MLW X FL & ML X FLW & \multirow{2}{*}{$\begin{array}{c}\text { Moyenne } \\
\text { des parents }\end{array}$} & \multicolumn{2}{c}{ Hétérosis } \\
\hline Taille moyenne de la portée & 7,5 & 9,75 & 11 & 8 & 8,63 & $0,28^{* *}$ & $-0,07^{\text {NS }}$ \\
Taux de mortinatalité (\%) & 16,67 & 10,26 & 22,73 & 6,25 & 13,46 & $0,69^{* * *}$ & $-0,54^{* * *}$ \\
Nés vivants (\%) & 83,33 & 89,74 & 77,27 & 93,75 & 86,54 & $-0,11^{*}$ & $0,08^{\text {NS }}$ \\
Mortalité naissance - sevrage (\%) & 16 & 20,00 & 15,79 & 8,33 & 18,00 & $-0,12^{*}$ & $-0,54^{* * *}$ \\
Taux de sevrage (\%) & 84 & 80,00 & 84,21 & 91,67 & 82,00 & $0,03^{\text {NS }}$ & $0,12^{*}$ \\
\hline
\end{tabular}

MLW X FL : mâle Large White X femelle locale ; ML X FLW : mâle local X femelle Large White, NS : P>0,05 ; ** : P<0,01 ; ***: P<0,001. 
Tableau 5 : Effet hétérosis des performances de croissance des porcs issus du croisement mâle Large White (MLW) et femelle locale (FL)

\begin{tabular}{lccccccc}
\hline \multirow{2}{*}{ Variables } & \multirow{2}{*}{ MLW } & \multirow{2}{*}{ FL } & Moyenne & \multicolumn{2}{c}{ MLW x FL } & \multicolumn{2}{c}{ Hétérosis } \\
\cline { 5 - 8 } & & & des parents & Mâle & Femelle & Mâle & Femelle \\
\hline P0 (g) & 845,45 & 491,43 & 668,44 & 883,33 & 739,3 & $0,32^{* * * *}$ & $0,11^{* *}$ \\
P15 (g) & 2800,4 & 900,5 & 1850,1 & 2235,3 & 1941,9 & $0,21^{* * *}$ & $0,05^{\text {NS }}$ \\
P30 (g) & 4181,8 & 1233,3 & 2707,6 & 4009,2 & 3761,5 & $0,48^{* * * *}$ & $0,39^{* * * *}$ \\
P60 (g) & 7023,1 & 1801,1 & 4400,5 & 6150,4 & 6246,1 & $0,40^{* * *}$ & $0,42^{* * * *}$ \\
P90 (g) & 9011,1 & 2520,2 & 5760,4 & 5401,5 & 5204,1 & $-0,06^{\text {NS }}$ & $-0,10^{\text {NS }}$ \\
\hline
\end{tabular}

Pi : Poids à i jour; MLW X FL : mâle Large White X femelle locale; MLW : mâle Large White ; FL: femelle locale ; ML : mâle local; FLW : femelle Large White; NS : P $>0,05$; ** : $\mathrm{P}<0,01$; *** : $\mathrm{P}<0,001$.

Tableau 6 : Effet hétérosis des performances de croissance des porcs issus du croisement mâle local (ML) et femelle Large White (FLW).

\begin{tabular}{cccccccc}
\hline \multirow{2}{*}{ Variables } & \multirow{2}{*}{ ML } & \multirow{2}{*}{ FLW } & \multirow{2}{*}{$\begin{array}{c}\text { Moyenne } \\
\text { des parents }\end{array}$} & \multicolumn{2}{c}{ ML x FLW } & \multicolumn{2}{c}{ Hétérosis } \\
\cline { 5 - 7 } & & & Mâle & Femelle & Mâle & Femelle \\
\hline P0 $(\mathrm{g})$ & 650,5 & 927,3 & 788,6 & 937,5 & 766,7 & $0,19^{* *}$ & $-0,03^{\text {NS }}$ \\
P15 $(\mathrm{g})$ & 1605,2 & 3313,6 & 2459,3 & 2675,2 & 2550,3 & $0,09^{\text {NS }}$ & $0,04^{\text {NS }}$ \\
P30 $(\mathrm{g})$ & 2533,3 & 4454,5 & 3493,9 & 4375,1 & 4083,3 & $0,25^{* * *}$ & $0,17^{* *}$ \\
P60 $(\mathrm{g})$ & 2800,2 & 7454,5 & 5127,3 & 7250,4 & 6366,7 & $0,41^{* * *}$ & $0,24^{* * *}$ \\
P90 $(\mathrm{g})$ & 4187,5 & 11785,7 & 7986,6 & 7775,2 & 6733,3 & $-0,03^{\text {NS }}$ & $-0,16^{* *}$ \\
\hline Pi : Poids à i jour : ML X FLW : mâle local X femelle Large White; MLW & : mâle Large White ; FL: femelle locale.
\end{tabular}

par l'éloignement de ces deux races sur le plan génétique. Ainsi, l'hétérosis a diminué la taille de la portée $(0,6 \%)$ et a augmenté légèrement le nombre de nés vivants à la naissance $(3,8 \%)$, à 14 jours $(9,1 \%)$, et au sevrage (28 jours, $8,7 \%)$ chez des races porcines voisines, notamment, le Yorkshire, le Landrace, le Large White et Chester White (Cassady et al., 2002). Une faible valeur de l'effet hétérosis a été également observée pour le poids à la naissance $(4,9 \%)$, à l'âge de 14 jours $(13,7 \%)$ et au sevrage (28 jours, $14,8 \%$ ) (Cassady et al., 2002). Ces effets augmentent également lorsque les croisements se font entre races génétiquement différentes (Hampshire, Duroc, Piétrain et Spot), avec des hétérosis de 36,$5 ; 38,3$; 51,9 et 51,9\%, respectivement pour la taille de la portée à la naissance, le nombre de nés vivants à la naissance, le nombre de nés vivants à 14 et à 28 jours d'âge (Cassady et al., 2002).

Par ailleurs, dans le cas d'un croisement terminal, l'effet hétérosis est plus important chez les descendants que dans le cas d'un croisement rotationnel (les femelles sont toujours choisies parmi les croisés et les mâles alternativement parmi les races parentales) et cette différence est de 0,$37 ; 0,30$ et 0,29 porcelets, respectivement pour la taille de la portée à la mise bas, le nombre de nés vivants et la taille de la portée à l'âge de 51 jours (Kuhlers et al., 1994). Les poids de la portée à la naissance et à 56 jours d'âge sont significativement plus élevés dans le croisement terminal que dans le croisement rotationnel (Kuhlers et al., 1994). Cette différence entre les deux modes de croisement s'explique par l'addition des effets hétérosis des parents (au moins trois) impliqués dans le schéma de croisement terminal. Par contre dans le cas d'un croisement rotationnel, deux parents sont le plus souvent impliqués et l'effet hétérosis est négligeable en F2 et dans les autres croisements qui suivent. Selon Kuhlers et al. (1994), la différence entre les effets hétérosis obtenus dans les schémas de croisement rotationnel et terminal est estimée à $14 \%$ pour les différents caractères de reproduction et de croissance chez le porc. Pour les performances de croissance, l'effet 
hétérosis des F1 est plus élevé que celui des backcross (Baas et al., 1992).

De manière générale, selon Bonnes et al. (1991), l'importance de l'effet d'hétérosis est fonction de plusieurs facteurs de variation. L'éloignement génétique entre les races permet d'avoir un effet hétérosis plus important comme il a été observé dans la présente étude. Dans le cas du croisement à double étage faisant intervenir une femelle elle-même croisée, l'hétérosis dû à l'utilisation d'une femelle croisée (hétérosis sur composante maternelle) et l'hétérosis dû au fait que les produits terminaux soient issus de croisements (hétérosis individuel ou hétérosis direct) sont cumulés. Dans le cas d'un croisement simple comme dans la présente étude, seul l'effet direct intervient. Les conditions de milieu peuvent aussi influencer l'hétérosis. Pour un caractère déterminé, il est en général, d'autant plus élevé que lorsque les animaux sont placés dans des conditions de milieu plus difficile. L'importance de l'hétérosis varie en fonction des caractères, comme leur héritabilité mais à l'inverse de celle-ci. D'une façon générale, on observe une opposition entre l'importance des effets additifs, caractérisée par l'héritabilité, et celle des effets non additifs des gènes, matérialisée par l'hétérosis. Cette opposition permet de prévoir le moyen le plus efficace d'amélioration génétique des performances. Il ne faut cependant pas en conclure que l'amélioration génétique résulte uniquement, selon les cas, de la « sélection en race pure » ou de la pratique des croisements. Il est en effet souvent préférable, pour obtenir l'efficacité maximum, d'associer sélection et croisement qui sont donc deux stratégies complémentaires et non concurrentes.

\section{Conclusion}

L'étude des performances zootechniques des porcelets issus du croisement entre Large White et la race locale a montré que le poids à la naissance et les gains moyens quotidiens des métis sont nettement supérieurs à ceux du porc local jusqu'au sevrage. La taille de la portée des truies croisées est supérieure à celle du porc local. Le croisement entre le porc de race locale et le Large White a amélioré la taille de la portée lorsque le mâle Large White est utilisé comme géniteur. Le croisement entre la race locale et la race Large White a également amélioré le poids des croisés par rapport aux locaux ; parmi les croisés, les meilleures performances de croissance ont été obtenues lorsque le mâle local est utilisé comme géniteur. Les différents croisements réalisés n'ont pas entraîné une différence pondérale entre les sexes. Au sein de la même race, les gains quotidiens moyens sont semblables et diffèrent entre races. L'effet hétérosis des performances pondérales et de paramètres de productivité numérique sont élevés. Les pourcentages de viabilité à la naissance et au sevrage ont peu varié d'une race à l'autre. L'effet hétérosis est négatif au niveau des pourcentages de mortinatalité et de la mortalité naissance-sevrage. Le croisement améliore les performances de reproduction et de production de la race locale du porc béninois lors du croisement avec le Large White.

\section{REFERENCES BIBLIOGRAPHIQUES}

Agbokounou AM. 2001. Etude des besoins énergétiques et protéiques du porc local béninois en phase de démarragecroissance. Mémoire de D.E.A. en Zootechnie, Faculté universitaire des sciences agronomiques de Gembloux, Belgique, 92.

Atodjinou FTR, Dotcho CDG. 2006. Caractéristiques de l'élevage des porcs locaux dans les élevages périurbains de Cotonou et d'Abomey-Calavi. Mémoire pour l'obtention du Diplôme des Etudes Agricoles Tropicales (D E A T), Lycée Meidji de Sékou, Bénin, 80.

Bass TJ, Christian LL, Rothschild MF. 1992. Heterosis and recombination effect in Hampshire and Landrace swine. Performance and carcass traits, Department of Animal Science, Iowa State University, Ames 50011, 70, 99105.

Bonnes G, Darré A, Fugit G, Gadoud R, Jussiau R, Manged B, Nadreau N, Papet A, Valognes R. 1991. Amélioration Génétique des Animaux d'Elevages. Collection INRAP ; 200.

Bonou D. 2006. Etude comparative de quelques performances zootechniques des porcs de race locale et métisse issue du croisement entre large white et landrace ; 
Mémoire d'Ingénieur des Travaux, Université d'Abomey-Calavi, Bénin, 63.

Cassady JP, Young LD, Leymaste KA, US. 2002. Heterosis and recombinaison effect on pig reproduction traits. Meat Animal Research Center, HRS, USDA, Claycenter, NE 68933-0166.

Codjo AB. 2003. Estimation des besoins énergétiques du porc local du Bénin en croissance entre 2 et $22 \mathrm{~kg}$ de poids vif. Tropicultura, 21(2) : 56-60.

D’Orgeval R. 1997. Le développement de l'élevage porcin en Afrique: l'analyse des systèmes d'élevage du porc local africain au Sud Bénin. Thèse de doctorat de l'Institut Nationale Agronomique, Paris Grignon, 273.

Devendra C, Fuller M. 1979. Pigs Production in Tropics. Edition de l'Université de Liège: Oxford University : London; 149.

Holnes HD. 1991. Le Porc. CTA Edition; 217.

Kuhlers DL, Jungst SB, Little JA. 1994. An experimental comparisen of equivalent terminal Rational crossbreeding system in swine. Sow and litter performance. Department of Animal and Dairy Science, Auburn University, Al 36849-5415, 72; 584-590.
Muys D, Westenbrink G, Meinderts J. 2003. L'élevage des porcs dans les zones tropicales. Série Agrodok $N^{\circ} 1$, Agromisa, Wagaeningen, 85.

Ramirez O, Tomas A, Clop A, Galmanomitogun O, Makuza Sm, Cadillo Jm, Kelly L, Armand Sanchez A, Amills M. 2006. Microsatellite and chromosome $\mathrm{Y}$ sequence analysis of wild boar and autochthonous pig breeds from Asia, Europe, South America and Africa. In Proceedings of the 30th International Conference on Animal Genetics, 2006, Porto Seguro, Brazil.: CBRA, 2006: Belo Horizonte, Brazil; 32.

SAS. 1991. User's Guide (Ressource Electronique) (4 ${ }^{\text {ème }}$ édn). SAS. Inst. Inc., SAS/STAT: Cary, version 6, New-York.

Youssao AKI. 2007. Cours d'Ethnologie des animaux productions. Département de Production et Santé Animales/EPAC/ Université Abomey-Calavi, Bénin, 90.

Youssao AKI, Koutinhouin GB, Kpodekon TM, Bonou AG, Adjakpa A, Ahounou GS, Mourot J. 2009. Performances zootechniques et aptitudes bouchères des porcs locaux au Sud du Bénin. Bull. Anim. Hlth. Prod. Afr., 57: 73-87. 